\title{
Capacitar y motivar a las niñas para su participación futura en el sector TIC. Propuesta de cinco países
}

\author{
Train and motivate girls for their future participation in the ICT sector. \\ Proposal from five countries
}

RECIBIDO 25/4/2019 ACEPTADO 29/12/2019 PUBLICAD0 1/12/2020

Carmen Fernández-Morante

Departamento Pedagogía y Didáctica, Universidad de Santiago de Compostela, España carmen.morante@usc.es

\section{(iD Beatriz Cebreiro López}

Departamento Pedagogía y Didáctica, Universidad de Santiago de Compostela, España beatriz.cebreiro@usc.es

\section{(iD) Lorena Casal Otero}

Departamento Pedagogía y Didáctica, Universidad de Santiago de Compostela, España lorena.casal@usc.es

\section{RESUMEN}

En la actualidad observamos que la brecha digital de género en cuanto al acceso, uso y formación en TIC (tecnologías de la información y la comunicación) está presente desde los primeros años de vida de los niños y las niñas, siendo la adolescencia la etapa en la que repercute sobre importantes decisiones futuras. En este artículo presentamos las aportaciones para la intervención educativa en la adolescencia, generadas en el marco del proyecto europeo «ICT Go Girls!», dirigidas a romper con los estereotipos de género en el uso de las TIC y conseguir que las niñas sean capaces de aprovechar futuras oportunidades de empleo en el sector TIC. Los resultados evidencian que hemos logrado romper con estereotipos y promover en las niñas una reflexión profunda sobre su futuro profesional. Con el Proyecto proporcionamos una metodología útil para que centros escolares y comunidades educativas puedan aplicar esta iniciativa de forma autónoma.

PALABRAS CLAVE competencia digital, género, educación secundaria, mujer, stem, metodología didáctica, estereotipos de género.

\section{ABSTRACT}

Currently we observe how the digital gender gap in access, use and training in Information and Communication Technologies (ICT) is present from the first years of life of boys and girls, being, adolescence, the stage where it affects important future decisions. . In this paper we present the contributions for educational integration in adolescence, generated within the framework of the European project “TIC Go Girls!", aimed at breaking with gender stereotypes in the use of ICTs and making girls capable of take advantage of employment opportunities in the ICT sector. The results show that we have managed to break with stereotypes and promote in girls a deep reflection on their professional future. With the Project we provide a useful methodology so that schools and educational communities have been this initiative in an autonomous way.

KEYWORDS digital competence/skill, gender, secondary education, girls, stem, didactic methods, gender stereotypes. 


\section{INTRODUCCIÓN}

La investigación sobre la participación de las mujeres en las TIC alerta de que la brecha digital de género persiste (Reinking y Martin, 2018; Verges, 2012) y constituye una barrera importante para la lograr la igualdad de oportunidades entre mujeres y hombres.

Castaño (2008) identifica varios niveles en la brecha de género que se mantienen en el tiempo:

1. Brecha del acceso a las TIC. El porcentaje de mujeres internautas está por detrás del de los hombres y su acceso es menos intenso, dado el menor tiempo de ocio, la tasa más baja de incorporación al mercado laboral o la ubicación en ámbitos laborales menos informatizados.

2. Brecha de formación. Las mujeres reciben una menor formación en lo que respecta a formación digital y capacidades. Además, aunque exista la posibilidad del acceso a Internet, esta no se rentabiliza si no se poseen habilidades para su uso.

3. Brecha de usos. Los usos de los hombres se relacionan más con el consumo y el ocio, mientras que las mujeres hacen un mayor uso relacionado con el bienestar social (formación, empleo e información sobre temas de salud).

Diferentes estudios constatan que el acceso, formación y uso de las TIC es diferente entre hombres y mujeres y, en esto, influyen los primeros años de vida de los niños y las niñas, cuando las niñas prefieren juegos al aire libre y los niños prefieren los videojuegos (Tatli, 2018). En este sentido, la investigación sugiere que la brecha digital de género se instaura desde una edad temprana, con diferentes expectativas e intereses para niños y niñas (Margolis y Fisher, 2002; Varma, 2010). Las ideas estereotipadas sobre género y tecnología digital se facilitan a través de juguetes y actividades de ocio específicos de género, de modo que los juegos relacionados con las tecnologías suelen estar dirigidos a niños mientras que juguetes más pasivos y afectuosos (por ejemplo, muñecas) suelen centrarse en niñas (Scantlebury y Baker, 2007).

Debido a que nuestro proyecto se contextualiza en los últimos años de la Educación Primaria y Primeros de la Secundaria, enumeramos algunas investigaciones que abordan la brecha de género en estas etapas educativas.

En un estudio realizado con alumnado del último ciclo de Educación Primaria (Casado et al., 2016), se demuestra que, aunque la informática resulta atrayente por igual a niños y niñas y que la creación de sus propias aplicaciones les resulta interesante, es en la percepción de su propia capacidad donde niños y niñas se ven diferentes, considerándose los niños en mayor medida más capaces que las niñas para hacer un programa informático.

Otro estudio con estudiantes de Educación Secundaria Obligatoria (Gargallo et al., 2003), concluyó que existe una brecha digital favorable a los adolescentes en lo concerniente a la disponibilidad, formación y uso. Sin embargo, las actitudes de las adolescentes son más favorables a la integración que las de los adolescentes.

En el estudio de Sánchez et al. (2012) se observa que las jóvenes poseen un alto sentimiento de competencia tecnológica, utilizan estrategias de autoaprendizaje y rechazan la educación formal en TIC. Las jóvenes que más usan las TIC se caracterizan por haber tenido un primer contacto temprano con los videojuegos, la edad de inicio varía entre los seis y ocho años. En ese mismo estudio, se confirma que la au- 
sencia de competidores a la hora de compartir el ordenador y la importancia que atribuye su familia al uso de herramientas tecnológicas, facilitaron que el videojuego superara el carácter lúdico y se convirtiera en un instrumento básico para su formación como usuarias avanzadas de las TIC.

En la etapa educativa de Bachillerato, la situación que recoge el estudio de Gil-Juárez et al. (2011) afecta a las elecciones de la formación y elección de perfil profesional, en la que las mujeres optan, en proporciones más bajas, por las asignaturas y/o los perfiles tecnológicos. En este sentido también podemos indicar que, como las decisiones con relación a la elección de los itinerarios formativos, se toman en la Educación Secundaria, el profesorado y las familias se convierten en agentes protagonistas y deberían ayudar a romper visiones estereotipadas de las profesiones y las tareas asociadas con ellas (Sáinz et al., 2016).

La influencia en la elección de profesión se refleja en los estudios superiores con la presencia hombremujer en las carreras. Son diversos los estudios que muestran que cada vez se matriculan más mujeres en las universidades del Estado español, pero estos mismos estudios también coinciden en señalar que la llegada no es uniforme en las diferentes áreas de conocimiento y que por lo tanto el incremento de mujeres no se traduce en una compensación de aquellas áreas con desequilibrios tradicionales de género (Gil-Juárez et al., 2012).

En España, solo el 17\% de estudiantes de informática son mujeres, una tasa similar al de resto de países de Europa (Sánchez et al., 2012). Si analizamos las estadísticas de elección de opciones académicas en el sistema universitario español observamos que siguen persistiendo las diferencias de género (Rodríguez et al., 2016). Este fenómeno no se da solo en España y la tendencia es similar en estudios en el ámbito internacional (Gil-Juárez et al., 2012). Así, la infrarrepresentación de las mujeres en el mundo TIC universitario, afirman Gil-Juárez et al. (2011) se hace especialmente visible tanto en la Unión Europea como en Estados Unidos. Las desigualdades horizontales de género parecen ser bastante estables. Las chicas terminan eligiendo más frecuentemente las profesiones de servicios "femeninos" y los chicos eligen trayectorias profesionales relacionadas con la ciencia, la tecnología, la ingeniería o las matemáticas (Hadjar y Aeschlimann, 2015). Este hecho dejará a las chicas fuera de las profesiones STEM (science, technology, engineering y mathematics), que será/es uno de los principales ámbitos laborales en el futuro.

Centrando nuestra atención en los motivos de esta situación, indicamos las causas enunciadas por Cussó (2014) en relación a las diferencias de género en el uso de las TIC:

1. Influencia de la familia y el profesorado.

2. Ausencia de modelos femeninos y permanencia de estereotipos asociados a un desconocimiento de las profesiones TIC.

3. Diferencia en las actitudes de chicos y chicas frente al uso de las TIC y de la autopercepción de sus habilidades.

En casa, los niños reciben más apoyo y aliento de las familias para usar tecnologías, mientras que el compromiso de las niñas con la tecnología tiende a ser más restrictivo y menos autónomo, lo que también puede limitar su oportunidad de explorar y desarrollar la curiosidad (Vekiri, 2013). En la escuela, aunque los docentes de primaria reconocen que desarrollar habilidades TIC es igualmente importante para todos los estudiantes, casi la mitad de ellos cree que es más probable que los niños tengan las características de aptitud, interés y personalidad para realizar estudios relacionados con las TIC (Vekiri, 2013). Además, el género del profesorado tiene impacto en las niñas ya que, como destacan Hadjar y Aeschlimann (2015), si las niñas 
son enseñadas por una maestra de matemáticas, ellas perciben la materia como un poco más femenina y muestran un mayor interés.

Todas las investigaciones consultadas indican que, independientemente del contexto de uso, existen diferencias entre géneros, tanto en acceso como en uso del ordenador. De manera consistente, en todas las investigaciones se comprueba que los niños invierten mucho más tiempo que las niñas en el uso del ordenador, tanto en casa como en la escuela o en las actividades extraescolares (García et al., 2012).

Con relación a la ausencia de modelos femeninos, Master et al. (2016) indican que el menor sentido de pertenencia de las niñas podría atribuirse a sentimientos de inferioridad con los estereotipos de la informática. Los autores, tras la realización de un estudio con adolescentes concluyen que, si se proporciona a las niñas un entorno educativo que no se ajusta a los estereotipos actuales de la informática, se aumenta su interés en las TIC y esto podría servir de base para intervenciones que ayuden a reducir las disparidades de género en la elección de carreras profesionales vinculadas con las TIC. En esta línea, la investigación de Lang et al. (2015) ha demostrado que el contacto o la exposición a expertos del mismo sexo beneficiará de forma sutil pero sistemática al autoconcepto de las niñas y, después de múltiples exposiciones o experiencias de contacto, este beneficio puede quedarse. Drury et al. (2011) afirmaron que maximizar la sensación de similitud percibida con los modelos a seguir es clave tanto para reclutar como para retener a mujeres en campos STEM.

Con relación a las actitudes, Verges (2012) indica que los niños tienen unas actitudes notablemente más positivas hacia los ordenadores, a los que encuentran más divertidos, importantes y amigables que las niñas. Y aún más: aunque la mayor parte de las niñas cree, en abstracto, que las mujeres pueden ser igual de competentes que los hombres en el uso del ordenador, cuando la pregunta hace referencia a ellas mismas como individuos, esta confianza decrece. Lang et al. (2015) indican que se fomenta una actitud más positiva por parte de las niñas cuando los planes de estudio incorporan el trabajo grupal o las asignaciones cooperativas en lugar de los proyectos individuales. Las niñas prefieren la colaboración a la competencia (Heemskerk et al., 2014).

En este contexto nace el proyecto Europeo «ICT Go Girls!»: Promoting Entrepreneurship among Secondary School Girls through ICT, integrado por siete instituciones europeas (Fernández-Morante y Cebreiro, 2013). El propósito del proyecto es intervenir de forma temprana, es decir, en el contexto escolar y en los primeros cursos de la educación secundaria (12-14 años) al considerar estas edades y niveles educativos, estadios clave en configuración de las identidades y en la definición de preferencias de estudios y profesiones.

En el proyecto «ICT Go Girls!» hemos diseñado, ejecutado y evaluado un programa piloto de intervención educativa que pretendía mejorar las habilidades digitales y de espíritu emprendedor entre las niñas de Educación Secundaria, utilizando las TIC como elemento clave. Esta iniciativa tuvo como objetivo proporcionar a las niñas conocimiento, habilidades y valores para ayudarlas a ser capaces de crear oportunidades de empleo en el ámbito de las TIC.

\section{OBJETIVOS DEL PROYECTO}

Con esta iniciativa pretendemos que las alumnas de Educación Secundaria tengan competencias digitales y de emprendimiento, así como un mayor conocimiento de las posibilidades laborales del sector TIC, para que sean conscientes de su valor como herramientas para su vida futura y para el empleo. 
Con «ICT Go Girls!» perseguimos los siguientes objetivos:

1. Desarrollar una metodología y un conjunto de recursos para las escuelas europeas, orientada a promover habilidades para el emprendimiento con TIC entre las alumnas de Educación Secundaria.

2. Colaborar con el profesorado y darles soporte en su actividad docente para que incorporen las habilidades TIC y para el emprendimiento en sus metodologías de aula.

3. Promover el desarrollo de competencias TIC entre las alumnas.

4. Mostrar el potencial de las TIC en los distintos ámbitos de la vida (escolar, laboral, personal...) para que lo tengan presente en sus futuras elecciones de estudios y profesiones.

5. Reforzar la cooperación entre los sectores educativo, de formación y del trabajo.

La finalidad del proyecto es proporcionar una metodología educativa y un conjunto de herramientas didácticas digitales para la intervención educativa en las escuelas europeas, permitiendo así la transferencia de conocimiento y promoviendo cambios para la igualdad de oportunidades que superen la brecha de género.

\section{METODOLOGÍA «ICT GO GIRLS!»}

La metodología «ICT Go Girls!» fue diseñada por un equipo de expertos de diferentes campos con la finalidad de ayudar a las alumnas de Educación Secundaria a cambiar estereotipos y pensamientos en torno a su vida, a reflexionar sobre su futuro profesional y conseguir que consideraren futuras opciones de estudio y laborales relacionadas con el ámbito de las TIC.

La metodología se validó en 5 países europeos (Alemania, Austria, Bélgica, España y Polonia). Es decir, la metodología se diseñó, se probó, se puso en marcha y se evaluaron los resultados obtenidos en esos 5 países. La metodología consta de cuatro fases que concluyen con una propuesta metodológica completa (Fernández-Morante y Cebreiro, 2013). La metodología siguió la siguiente secuencia:

1. Búsqueda y análisis de buenas prácticas en materia de Mujer, emprendimiento y TIC. Se analizaron un total de 40 proyectos exitosos puestos en marcha en diferentes países.

2. Elaboración de la Metodología «ICT Go Girls». Trabajo liderado por el Grupo de Tecnología Educativa de la Universidad de Santiago de Compostela. Teniendo en cuenta las lecciones aprendidas del análisis de los 40 ejemplos de buenas prácticas analizadas y la realidad educativa de los 5 países participantes en el estudio piloto, se diseñó la propuesta metodológica. Dicha propuesta promovía actitudes positivas hacia las tecnologías, el desarrollo de competencias TIC y la creación de iniciativas emprendedoras en las escuelas de Educación Secundaria. La metodología didáctica se desarrolló en un entorno tecnológico con una plataforma de red social que soportaba todo el proceso presencial y virtual para permitir un proceso de formación continuo que soporta al aprendizaje conjunto, el aprendizaje individualizado con un portfolio, la interacción en distintos niveles (grupos pequeños, grupos por país y el grupo completo de todos los participantes) y las creaciones de los participantes. Este desarrollo metodológico, que se presentará en detalle más adelante, abordó a través de diferentes actividades de aula, las ideas sobre los roles profesionales y perspectiva de género (socialización 
previa del alumnado de secundaria). Se completó, además, el proceso educativo con el análisis e interacción directa del alumnado de secundaria con mujeres líderes, empresarias y expertas en TIC. Esta última fase pretendía mostrar ejemplos de mujeres que son referentes en profesiones vinculadas con las TIC con la finalidad de animar a las alumnas a optar por estudios y profesiones TIC.

3. Desarrollo del estudio piloto. Se llevó a cabo en 10 centros educativos europeos de Educación Secundaria de 5 países ( 2 por país) y con un mínimo de 10 alumnas por centro. Se trabajó con todo el alumnado de las aulas (chicas y chicos), centrándonos especialmente en ellas como líderes de los grupos.

4. Evaluación de los resultados. En el estudio piloto se recogieron evidencias a través de los participantes (profesorado, alumnado y coordinadores del proyecto en cada país).

La metodología, diseñada para ser implementada en los primeros cursos de la Educación Secundaria, facilita al profesorado un procedimiento de trabajo flexible con posibilidades de adaptación a las necesidades propias de cada aula. Permite trabajar las TIC de una forma motivadora y propicia, en las alumnas de esta etapa, un mayor interés y conocimiento encaminado a la toma de decisiones vinculada a la elección de estudios tecnológicos.

La metodología se divide en dos etapas en las que se desarrollan diferentes actividades:

\title{
TABLA 1. Metodología «ICT Go Girls»
}

ETAPA 1. Socialización previa: ideas sobre los roles profesionales y la perspectiva de género.
ETAPA 2. Modelos profesionales: mujeres líderes, empresarias y expertas en TIC.
Actividad 0. Punto de partida: Pensando en mi futuro profesional ¿A qué me gustaría dedicarme profesionalmente en el futuro?
Actividad 4. Estudio de casos: Mujeres con una trayectoria profesional exitosa vinculada a las TIC.

Actividad 1. Presentación del proyecto Actividad 5. Proyecto Empresarial: Creando tu empresa virtual.

Actividad 2. Tecnología: ¿Cómo y para qué utilizamos las tecnologías?
Actividad 6. Actividad Final. Pensando en mi futuro profesional: Los perfiles profesionales y mis preferencias laborales.

Actividad 3. Profesionales y empresas: Profesiona-

les y empresas que usan las TIC presentes en su comunidad.

\author{
Cada una de las actividades incluye: \\ -Objetivos para el proyecto. \\ -Objetivos para el docente. \\ -Tarea (descripción, lugar, medio, material didáctico, \\ rol del coordinador, rol del docente, dinámica del aula y observaciones).
}


Con estas actividades se pretende que las niñas tengan un plan de carrera que les ayude en la transición desde la Educación Secundaria a la entrada en una opción de carrera universitaria o empresarial en el sector de las TIC. Las actividades se dirigen a trabajar la autoconciencia de las niñas, romper estereotipos de género en el uso de las TIC y a promover la reflexión sobre el futuro profesional para facilitar el pensamiento profundo sobre los nichos de empleo emergentes y el valor estratégico de las TIC.

\section{PRODUCTOS GENERADOS}

El proyecto ha generado una serie de materiales prácticos para escuelas de Europa con la finalidad de ayudar a su personal a reflejar, aplicar y adaptarlo a las necesidades individuales. Se generaron los siguientes materiales:

1. Informe de investigación sobre las iniciativas y recomendaciones europeas previas.

2. Metodología educativa, paso a paso, para la promoción de las TIC y del espíritu emprendedor en las escuelas europeas. Permite fomentar las TIC y estimular el espíritu emprendedor en las jóvenes, con el objetivo principal de fomentar entre las generaciones jóvenes ser más abiertos y flexibles a la hora de tomar decisiones sobre su carrera.

3. Plataforma social, de software libre, basada en Elgg y SocialWire, para la comunicación interactiva y la creación de redes entre escuelas y alumnado de los países europeos.

4. Manual de uso de la plataforma, desarrollado específicamente para las escuelas y el profesorado en Europa.

5. Manual para centros educativos. Es una guía práctica dirigida a escuelas, educadores y administraciones educativas que además de contextualizar e introducir el proyecto, presenta la metodología que se desarrolló, los materiales generados, un detalle de las actividades llevadas a cabo y las conclusiones alcanzadas. El objetivo del manual es evidenciar cómo las alumnas de primer ciclo de Educación Secundaria en Europa pueden llegar a comprometerse con materias relacionadas con las TIC y desarrollar el espíritu emprendedor, con el objetivo final de que puedan valorar diferentes posibilidades de estudios superiores y de carreras profesionales con TIC. La identificación de ejemplos de buenas prácticas a partir de la experimentación llevada a cabo en los países socios describe lo que hay que hacer y cómo abordar el tema de las jóvenes y las TIC desde diferentes perspectivas y en diferentes regiones.

También se elaboraron una serie de videomensajes animando a las chicas a interesarse en las TIC. En los vídeos, diferentes mujeres con relevancia en el ámbito de las TIC animan a las chicas a considerar la informática en su futuro laboral y de estudios, y cuentan, además, cómo iniciaron ellas su trabajo en este campo y se puede visionar en el portal del proyecto.

\section{RESULTADOS}

Como ya hemos comentado, uno de los productos del proyecto ha sido el "Manual para Centros Educativos" (Rodríguez et al., 2014). En este manual, que puede consultarse en: https://e-learning.cesga.es/ictgogirls/ 
proyecto/productos/, se exponen, entre otros, los resultados, conclusiones y lecciones aprendidas durante el tiempo de realización del proyecto. Nosotros, para este artículo hemos decidido presentar algunos de los resultados más relevantes.

Durante la fase de pilotaje, detectamos, en línea con investigaciones en el campo (García et al., 2012; Heemskerk et al., 2009; Lang et al., 2015; Lasen, 2010), que es necesario que las alumnas de Educación Secundaria de Europa se vean a sí mismas con posibilidades y con capacidades para insertarse en un entorno laboral en el que las TIC son las protagonistas. Pudimos observar cómo el proyecto contribuía a la motivación de las niñas ya que, tras realizar diferentes actividades, fueron capaces de considerar, como posibles opciones laborales de futuro, diferentes profesiones relacionadas con las TIC. El pilotaje demostró que las jóvenes pensaban, antes de la realización de las actividades, que un empleo menos cualificado sería más sencillo de alcanzar para ellas. Sin embargo, las alumnas comprendieron que pueden desarrollar su actividad laboral futura en un entorno con TIC y que las mujeres formadas pueden ser un gran un activo para las empresas. Este tipo de sensibilización contribuye a que más mujeres se involucren en el desarrollo de competencias TIC y en su formación, además de mejorar en los procesos de toma de decisiones. Dado que en la actualidad solo un pequeño porcentaje de mujeres opta por las TIC, y rara vez alcanzan puestos directivos, la iniciativa representa una gran medida para contribuir al proceso de cambio.

Comparando los resultados de pilotaje de los países socios, pudimos confirmar que en el inicio de la etapa de Educación Secundaria la mayoría del alumnado muestra, en general, interés por las TIC. Alrededor de esta edad (12 años) todavía no son conscientes del potencial que puedan tener en su educación y carreras futuras, pero disfrutan usando las tecnologías tanto en casa como, si se les da la oportunidad, en la escuela. Se demostró que en las clases prácticas el uso de ordenadores de una manera menos tradicional puede aumentar la motivación general hacia las TIC e influir en las alumnas en el momento de decidir qué estudios superiores elegir. El hecho de que se les diera la opción de usar los ordenadores y otros equipos "de una manera diferente" en el ambiente escolar, fue muy apreciado, ya que, además, se percibe como "divertido". En este sentido, la investigación en el campo incide en que las niñas aprecian las instrucciones paso a paso y una función de ayuda más clara que los niños. Además, muchos autores mencionan la importancia de una retroalimentación clara e inmediata en el trabajo con las niñas (Heemskerk et al., 2009).

El formato de red social elegido fue un éxito por la proximidad de los jóvenes de estas edades con este tipo de herramientas digitales. La plataforma social desarrollada durante el proyecto se utilizó como una herramienta interactiva para la subida y descarga de archivos y de intercambio de información; percibiéndose como un pre-escenario del entorno de trabajo en el sector TIC. Era necesario involucrar a las alumnas en las tareas técnicas para que se enfrentaran a las herramientas de forma natural y superaran los miedos iniciales. Las jóvenes podían expresar sus opiniones en las aulas y discutir con el profesorado en igualdad de condiciones. Esto incrementó la autoestima de muchas niñas y, en particular, la de las jóvenes en situaciones más vulnerables. Otro logro importante fue la cantidad de trabajo creativo realizado y el efecto positivo del trabajo en equipo.

La implicación de las jóvenes en el pilotaje fue imprescindible para el éxito del proyecto «ICT Go Girls», tanto por ser de gran utilidad práctica como porque ha demostrado ser crucial en la mejora del proceso de toma de decisiones. Se pudo observar que el proyecto fue considerado una herramienta de gran alcance para apoyarlas y proporcionarles información profesional adecuada en la edad y el momento preciso. 
Uno de los elementos que más se valoró en el pilotaje de la metodología fue el uso de herramientas divertidas y creativas para trabajar en las diferentes actividades educativas. Tanto profesorado como estudiantes manifestaron que las herramientas utilizadas para crear infografías multimedia y carteles, o la programación con Scratch, así como algunos dispositivos entretenidos, tales como Makey Makey o Lego WeDo, resultaron muy atractivos para chicos y chicas. Por otra parte, fue motivadora la interacción con escuelas y alumnado de otros países europeos.

El pilotaje también demostró, en línea con la investigación generada en los últimos años (Drury et al., 2011; Lang et al., 2015) que las alumnas apreciaban la posibilidad del contacto directo con los modelos de referencia presentados (mujeres exitosas); en particular, aquellos contactos que les permitían hablar o comunicarse, ya sea en persona, por correo electrónico o videoconferencia. Los modelos a seguir eran ejemplos reales con los que podían relacionarse e intercambiar opiniones, así como ayudar a aclarar dudas. El proyecto demostró que las alumnas podían aprender, tanto de las mujeres que tienen éxito y ya han subido en el escalafón laboral, como de las mujeres más jóvenes que acababan de comenzar a trabajar en el campo de las TIC como empresarias y que pueden compartir sus preocupaciones, pasiones y su vida personal con las estudiantes. La vida familiar futura, la maternidad y cómo combinar esto con el trabajo, sobre todo en un sector dominado por los hombres, resulta de gran relevancia para las chicas y su futura elección de carrera. El contacto personal con estas mujeres fue una forma muy positiva y eficaz de influir en las alumnas.

Otros aspectos observados durante el pilotaje fueron los siguientes:

- Es necesario utilizar un lenguaje de género y evitar los estereotipos en imágenes, storyboards, etc., especialmente cuando se trata de sectores con brecha de género y estereotipos profundamente arraigados. El profesorado tiene que reflexionar cuidadosamente sobre los mensajes que están dando y promover la coeducación con perspectiva de género.

- La seguridad de Internet juega un papel importante y las familias, profesorado y alumnado necesitan estar informados sobre todos los aspectos, especialmente la seguridad de los datos personales y el uso correcto de los medios de comunicación social.

- Las jóvenes más vulnerables afrontan más retos que sus iguales y necesitaron más explicaciones y ayuda del profesorado. Este mayor esfuerzo es necesario con el fin de trabajar con ellas y ayudarlas a alcanzar un nivel apropiado. Al inicio, estas niñas optaban más por profesiones típicamente femeninas (peluquera, cocinera, etc.) pero, tras el trabajo con el profesorado, tenían una perspectiva más amplia sobre posibles trabajos que podrían desarrollar en su futuro laboral. Algunas alumnas no percibían inicialmente las ventajas y desventajas de las diferentes profesiones en el sector de las TIC, pero después del trabajo práctico en el aula quedó mucho más patente y se puede decir que se logró una influencia positiva en cuanto a la elección de la profesión.

Durante el estudio se constató la influencia que las escuelas, el profesorado y la familia, ejercen sobre las niñas para ayudarlas a mejorar su potencial de liderazgo y animarlas a optar por una mayor implicación en el sector de las TIC. Este resultado se relaciona con otros en el campo (Abbiss, 2011; Casado et al., 2016; Margolis y Fisher, 2002; Sánchez et al., 2012; Vekiri, 2013).

Para finalizar la presentación de los resultados nos gustaría destacar la importancia de la familia, ya que es la instancia clave en la socialización de todas las personas y conforma un espacio -e institución-de enorme poder, entendido este en términos de influencia psicológica, educativa, social y emocional donde 
confluyen múltiples variables, tanto estructurales como de índole dinámica (Leiva Olivencia, 2016). Comprender la interacción de los factores de género y familia que determinan las aspiraciones de carrera de los adolescentes en los campos STEM parece ser crucial para diseñar intervenciones significativas y efectivas basadas en la escuela y la familia (Sáinz y Müller, 2018).

Las alumnas pudieron aprender en este proyecto quiénes son como ciudadanas y cómo sus experiencias en la escuela están conectadas con el éxito futuro. Fueron capaces de visualizar el camino por el que los condicionamientos sociales, sus tendencias y su bagaje cultural influyen en sus creencias y determinan quiénes son. Además, pudieron analizar por qué el trabajo es importante para ellas y qué papel debería desempeñar en su vida o por qué las decisiones actuales influyen en su futuro. También aprendieron a comunicar en casa posibles razones para optar por una carrera TIC y las ventajas que puede suponer convertirse en emprendedoras en el sector de las tecnologías en comparación con otras vías. Gran parte del profesorado involucrado observó enormes progresos que demostraron la eficacia de la metodología «ICT Go Girls».

\section{DISCUSIÓN Y CONCLUSIONES}

Los resultados obtenidos en nuestro estudio confirman que la brecha digital de género está presente y que es la última etapa de Educación Primaria y la Educación Secundaria el momento propicio para realizar una intervención educativa que ayude a las niñas a romper estereotipos y conseguir que sean capaces de aprovechar futuras oportunidades laborales en el sector TIC. Además, encontramos en nuestra investigación que la familia y los docentes son agentes fundamentales para la ruptura de esta brecha digital de género y, como hemos podido comprobar, nuestros resultados están en línea con otros trabajos que también han puesto de manifiesto esta relación (Abbiss, 2011; Lepinik y Samec, 2013; Sánchez et al., 2012; Tiainen y Berki, 2019; Vekiri, 2013). No podemos decir que los resultados de nuestro trabajo sean inesperados, ya que, como hemos podido comprobar, hay suficiente evidencia empírica acumulada que constata el impacto positivo de una intervención educativa adecuada.

Las conclusiones que pudimos obtener con la realización es este trabajo apuntan, principalmente, en tres direcciones: de carácter más conceptual, de carácter metodológico y de carácter político-institucional.

Con relación a la primera, tenemos que indicar que la relación de las niñas y las TIC se ve muy marcada por las percepciones y creencias que tienen con relación a las TIC. Las niñas continúan mostrando una menor confianza en sus habilidades digitales que los niños (Lasen 2010). Para algunos, la imagen es la clave y es necesario romper los mitos y estereotipos actuales. Otros ven que es un "problema" institucionalizado, con escuelas y maestros malinterpretando capacidades masculinas y femeninas (Miliszewska y Moore, 2011). Aumentar la confianza de las niñas en sí mismas es primordial para que contrarresten las dudas de género al trabajar con TIC (Heemskerk et al., 2014).

Con relación a la segunda, el proyecto «ICT Go Girls» propone una intervención educativa innovadora para que las niñas se autoperciban capaces para usar las tecnologías en igualdad a los niños y las consideren un potente instrumento en su desarrollo personal, profesional y social. La base de la metodología se centra en actividades en las que las niñas se sienten protagonistas y en las que trabajan de forma colaborativa.

Las actividades «ICT Go Girls» se planificaron inicialmente para realizarse en el contexto de clase, como una iniciativa transversal que podría ser trabajada en diferentes tipos de materias escolares. Sin embargo, la realidad demostró que en algunas escuelas piloto esto presentaba dificultades de integración en la pla- 
nificación del centro. Por lo tanto, se hacía necesario ser flexible con la propuesta, el ajuste de los tiempos y las actividades a la realidad concreta de las escuelas participantes. A pesar de estas limitaciones, el profesorado observó que el pilotaje contribuyó a un cambio de actitud, al desarrollo de competencias y a la mejora de la autoestima de las alumnas.

También tenemos que indicar que el apoyo al profesorado plantea la cuestión de cuánto tiempo y esfuerzo hace falta para desarrollar un nuevo conjunto de destrezas y cómo deben ser reconocidas oficialmente. Una forma de hacerlo es asegurarse que las actividades de formación permanente del profesorado reconozcan este tipo de iniciativas y esfuerzos en el marco de la educación permanente del profesorado. Siendo, desde nuestro punto de vista la opción principal, si es posible, su integración dentro del currículum también podría integrarse en las actividades extra-escolares, proyectos específicos semanales, clubes escolares, etc.

El proyecto ha proporcionado una metodología útil y un paquete de herramientas completo (con materiales de apoyo, metodologías y software) para que los centros escolares y las comunidades educativas puedan aplicar esta iniciativa en otras regiones de Europa. El proyecto «ICT Go Girls» tiene una visión a largo plazo, con la seguridad de que veremos más empresas dirigidas por mujeres en el futuro, pero para ello es necesario un mayor apoyo a las mujeres en sus responsabilidades adicionales y en la conciliación de la vida familiar y laboral.

Con relación a la tercera, algunas de las lecciones aprendidas durante la implementación del proyecto nos invitan a reflexionar sobre la necesidad de políticas públicas de apoyo a las iniciativas educativas innovadoras y al desarrollo de competencias TIC en edades tempranas (alfabetización digital), siendo necesario implementar las competencias básicas en TIC de forma gradual. Existe un déficit en la competencia digital docente para que el profesorado pueda integrar de forma adecuada estos recursos (Arabit y Prendes, 2019) y, para ello resultan esenciales las estrategias de apoyo al profesorado y a los y las jóvenes con el fin de hacer un uso más eficaz de las TIC. Los esfuerzos individuales del profesorado son limitados si no cuentan con el apoyo institucional de las autoridades escolares, la dirección del centro y los responsables y autoridades educativas. Centrarse en una mejor colaboración entre las autoridades escolares, dirección de escuelas, profesorado, familias y alumnado es de gran importancia a la hora de introducir nuevas formas de trabajar en las aulas; con el fin de ayudar a las chicas jóvenes a interesarse y desarrollar una carrera profesional en el sector TIC.

A la luz de los resultados de este estudio podemos concluir que los esfuerzos para conseguir hacer más atractiva la profesión de informática a niños y niñas, deberían iniciarse en la última etapa de la enseñanza primaria y concretarse en la Educación Secundaria. Además, en el caso particular de las niñas, este esfuerzo debería orientarse a la motivación y confianza en sus capacidades para la programación de cualquier aplicación.

\section{APOYOS}

Este artículo ha sido realizado en base a la investigación llevada a cabo desde el Proyecto «ICT Go Girls!» Promoting Entrepreneurship amomg secondary school girls through ICT. «ICT Go Girls!» nace como un proyecto de investigación competitivo financiado por la Comisión Europea en el marco de la acción COMENIUS del Lifelong Learning Program (2012). Número de referencia del Proyecto: 526590-LLP-1-2012-1-ES-COMENIUS-CMP. 


\section{REFERENCIAS}

Abbiss, J. (2011). Boys and machines: Gendered computer-identities, regulation and resistance. Gender and Education, 23, 601-617. https://doi.org/10.1080/09540253.2010.549108

Arabit, J., y Prendes, M. P. (2019). Metodologías y Tecnologías para enseñar STEM en Educación Primaria: análisis de necesidades. Píxel-Bit. Revista de Medios y Educación, 57, 107-128. https://doi.org/10.12795/pixelbit.2020.i57.04

Casado, C., Meneses, J., y Sancho, T. (2016). ¿Cómo ven los alumnos de primaria la profesión informática? Influencia del género y la percepción de su capacidad. Píxel-Bit. Revista de Medios y Educación, 49, 149-161. http://dx.doi.org/10.12795/ pixelbit.2016.i49.010

Castaño, C. (Dir.) (2008). La Segunda Brecha Digital. Cátedra.

Cussó, R. (2014). Género y actitudes ante las TIC: estudio de la influencia del uso de ordenadores personales en los centros escolares. http://repositori.udl.cat /bitstream/handle/10459.1/56849/rcussoc.pdf?sequence=1\&isAllowed=y

Drury, B. J., Siy, J. O., y Cheryan, S. (2011). When do female role models benefit women? The importance of differentiating recruitment from retention in STEM. Psychological Inquiry: An International Journal for the Advancement of Psychological Theory, 22, 256-269. https://doi.org/10.1080/104784 0X.2011.620935

Fernández-Morante, C. y Cebreiro, B. (2013). ICT Go Girls!: promoviendo el emprendimiento con TIC entre las alumnas de secundaria. En J. Sánchez, J. Ruiz y E. Sánchez (Coords.), Buenas prácticas con TIC en la investigación y la docencia. Universidad de Málaga.

García, I., Gros, B., y Escofet, A. (2012). La influencia del género en la cultura digital del estudiantado universitario. Athenea Digital, 12(3), 95-114. https://doi.org/10.5565/rev/athenead/ v12n3.1075

Gargallo, B., Suárez, J., y Belloch, C. (2003). La división digital en el proceso de integración de las NTIC en la educación. Diferencias de género entre alumnos de E.S.O. de la comunidad valenciana. Teoría de la Educación, 4(1). http://dx.doi. org/10.14201/eks.14343
Gil-Juárez, A., Vitores, A., Feliu, J., y Vall-Llovera, M. (2011). Brecha digital de género: Una revisión y una propuesta. Education In The Knowledge Society (EKS), 12(2), 25-53.

Hadjar, A., y Aeschlimann, B. (2015). Gender Stereotypes and Gendered Vocational Aspirations among Swiss Secondary School Students. Educational Research, 57(1), 22-42. https:// doi.org/10.1080/00131881.2014.983719

Heemskerk, I., Geert ten Dam, Volman, M., y Admiraal, W. (2014). Gender Inclusiveness in Educational Technology and Learning Experiences of Girls and Boys. Journal of Research on Technology in Education, 41(3). https://doi.org/10.1080/1539 1523.2009.10782531

Lang, C., Fisher, J., Craig, A., y Forgasz, H. (2015). Outreach programmes to attract girls into computing: how the best laid plans can sometimes fail. Computer Science Education, 25(3), 257-275. 10.1080/08993408.2015.1067008

Lasen, M. (2010). Education and career pathways in information communication technology: What are schoolgirls saying? Computers \& Education, 54, 1117-1126. https://doi. org/10.1016/j.compedu.2009.10.018

Leiva Olivencia, J. (2016). Santos Rego, M. Á. (ed.) (2015) El poder de la familia en la educación. Madrid, Editorial Síntesis. Teoría de la Educación, 28(1), 259-262. http://revistas.usal.es/ index.php/1130-3743/article/view/14778/15291

Lepinik, J., y Samec, P. (2013). Uso de tecnologías en el entorno familiar en niños de cuatro años de Eslovenia. Comunicar: Revista científica iberoamericana de comunicación y educación, 40, 119-126. http://dx.doi.org/10.3916/C40-2013-03-02

Margolis, J., y Fisher, A. (2002). Unlocking the clubhouse: Women in computing. MIT Press.

Master, A., Cheryan, S., y Meltzoff, A. (2016). Computing Whether She Belongs: Stereotypes Undermine Girls' Interest and Sense of Belonging in Computer Science. Journal of Educational Psychology, 108(3), 424-437. http://dx.doi.org/10.1037/ edu0000061

Miliszewska, I., y Moore, A. (2011) Attracting Girls to ICT studies and careers: A snapshot of Strategies. Redress: Association of Women Educators, 20(3) 10-14. 
Reinking, A., y Martin, B. (2018). The Gender Gap in STEM Fields: Theories, Movements, and Ideas to Engage Girls in STEM. Journal of New Approaches in Educational Research, 7(2), 148153. http://dx.doi.org/10.7821/naer.2018.7.271

Rodríguez, M. J., Ilijic, B., Fernández-Morante, C., Cebreiro, B., y Wolf, B. (2014). Manual para Centros Educativos. Promoviendo el espíritu emprendedor a través de las TIC. www.cesga.es/ es/noticias/descargaDocumento/id/193

Rodríguez, M., Peña, J., y García, O. (2016). Estudio cualitativo de las diferencias de género en la elección de opciones académicas en los estudiantes del bachillerato científico-técnico. Teoría de la Educación, 28(1), 189-207.

Sáinz, M. y Müller, J. (2018). Gender and family influences on Spanish students' aspirations and values in stem fields. International Journal of Science Education, 40(2), 188-203. https:// doi.org/10.1080/09500693.2017.1405464

Sáinz, M., Meneses, J., López, B., y Fàbregues, S. (2016). Gender Stereotypes and Attitudes Towards Information and Communication Technology Professionals in a Sample of Spanish Secondary Students. Sex Roles. A Journal of Research, 74(34), 154-168.

Sánchez, N., Ortega, O., y Vall-Llovera, M. (2012). Romper la brecha digital de género. Factores implicados en la opción por una carrera tecnológica. Athenea Digital, 12(3), 115-128. https://doi.org/10.5565/rev/athenead/v12n3.1133

Scantlebury, K., y Baker, D. (2007). Gender issues in science education research: Remembering where the difference lies. En S. Abell y N. Lederman (Eds.), Handbook of research on science education (pp. 257-286). Lawrence Erlbaum.

Tatli, Z. (2018). Traditional and Digital Game Preferences of Children: A CHAID Analysis on Middle School Students. Contemporary Educational Technology, 9(1), 90-110.

Tiainen, T., y Berki, E. (2019). The re-production process of gender bias: a case of ICT professors through recruitment in a gender-neutral country. Studies in Higher Education, 44(1), 170184. https://doi.org/10.1080/03075079.2017.1351428

Varma, R. (2010). Why so few women enroll in computing? Gender and ethnic differences in students' perception. Computer Science Education, 20, 301-316. https://doi.org/10.1080/0899 3408.2010 .527697
Vekiri, I. (2013). Information science instruction and changes in girls' and boys' expectancy and value beliefs: In search of gender-equitable pedagogical practices. Computers \& Education, 64, 104-115. https://doi.org/10.1016/j.compedu.2013.01.011

Verges, N. (2012). De la exclusión a la autoinclusión de las mujeres en las TIC. Motivaciones, posibilitadores y mecanismos de autoinclusión. Athenea Digital, 12(3), 129-150. https://doi. org/10.5565/rev/athenead/v12n3.1042 